\title{
An Unusual Presentation of Corneal Perforation in a Neonate Following Instrument-Assisted Delivery
}

\author{
Christolyn Raj', John Ruddle', Alice Downey² \\ ${ }^{1}$ Department of Ophthalmology, Royal Children's Hospital, Melbourne, Australia \\ ${ }^{2}$ Department of Obstetrics \& Gynecology, Launceston, Tasmania \\ Email: ChristolynRaj@rch.org.au
}

Received 22 May 2014; revised 21 June 2014; accepted 17 July 2014

Copyright (C) 2014 by authors and Scientific Research Publishing Inc.

This work is licensed under the Creative Commons Attribution International License (CC BY). http://creativecommons.org/licenses/by/4.0/

c) (i) Open Access

\section{Abstract}

A 25-week-old neonate was noted to have unilateral corneal edema and buphthalmos at birth following a forceps assisted delivery. Ten days later, examination of the neonate under anesthesia showed a central corneal perforation with a possible retinal detachment. By this time the neonate's affected eye was deemed unsalvageable, and therefore an enucleation was performed with insertion of an ocular implant. Subsequent histopathology of the enucleated eye revealed a grossly malformed anterior segment and findings usually associated with congenital glaucoma. This is the first reported case in the literature of a neonatal corneal perforation in the context of instrument-assisted delivery. This case highlights the need to actively investigate the aetiology of cloudy corneas in neonates. Early exploration and surgical intervention may be necessary especially if congenital glaucoma is suspected to be the underlying aeitlogy.

\section{Keywords}

Corneal Perforation, Congenital Glaucoma, Birth Trauma, Enucleation

\section{Case Report}

A 25 weeks old preterm baby (46XY, weight 1032 grams, Apgar score at birth of 4 at 1 min and 6 at 5 mins) was delivered by forceps-assisted instrumentation. At (35 weeks +4 days) he was referred to the ophthalmologist for review of right-sided unilateral bupthalmos and diffusely hazy cornea. The cornea in the contralateral eye was clear with a normal appearing iris and anterior chamber. The intraocular pressures were $22 \mathrm{mmhg}$ in right eye and 15 mmhg in left eye (Figure 1). The right retina could not be visualized due to the hazy cornea. In 
the left eye the retina showed immature retinal vascularisation in keeping with the degree of prematurity of the neonate.

Ten days post delivery, the right corneal haze increased and showed a central area of perforation. An ultrasound B-scan performed at that time indicated a funnel-shaped retinal detachment in the right eye. An exploration under anesthesia was subsequently performed and revealed a central corneal perforation with a disorganized anterior segment and loss of the native intraocular lens. A decision to enucleate the right eye was made and a bioceramic implant (ocular prosthesis) was fitted 8 weeks later with excellent cosmesis.

The left eye continues to develop normally.

Histopathology of the enucleated eye indicated a corneal perforation with subsequent fibrovascular scarring as well as structural malformation of the outflow drainage channel and features associated with primary congenital glaucoma (Figure 2, Figure 3).

\section{Discussion}

Neonatal corneal perforation is a rare occurrence [1]. Instrumentation-assisted deliveries have been reported to cause minor ocular trauma including peri-orbital bruising, subconjunctival hemorrhage, corneal abrasions and

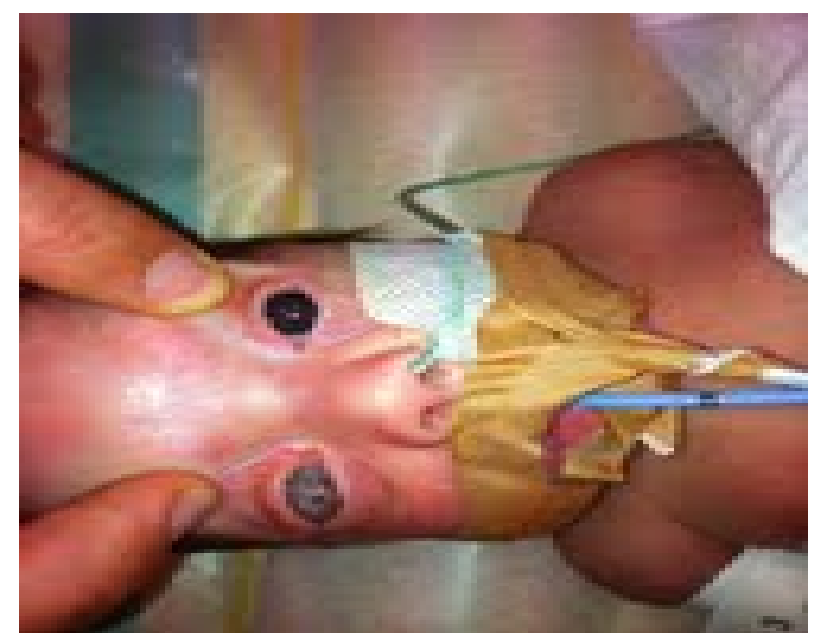

Figure 1. 25-week-old ex-premature neonate with right eye corneal edema and bupthalmos.

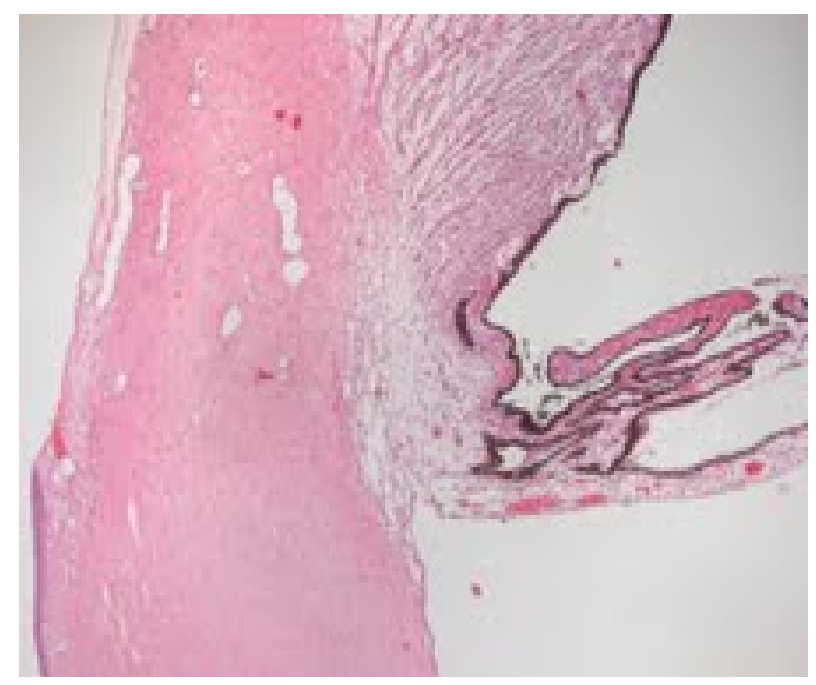

Figure 2. Medium power vew H/7E stain: anbnormal anterior insertion of the iris root into the trabecular meshwork. 


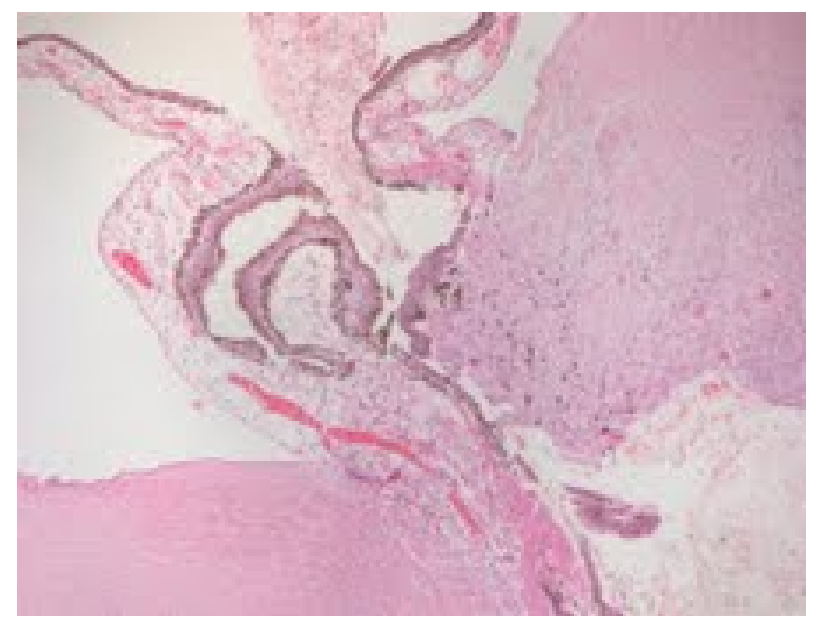

Figure 3. Medium power view H\&E stain: focal adhesion of the iris to the area of corneal scarring which may represent anterior segment malformation prior to corneal perforation.

tears in Descemet's membrane. Whilst the occurrence of a corneal or globe perforation in the setting of birth related traumatic delivery is plausible, there are no reported cases in the literature.

In most cases ocular trauma during delivery may initially present with a hazy cornea which improves over weeks. Failure to improve should raise the suspicion of other possible causes (Table 1).

Birth trauma was initially entertained as the most likely explanation for corneal perforation in this neonate because of the clinical features of the neonate at birth which included extensive facial bruising and intra-parenchymal hemorrhages. However it must be kept in mind that these are often expected features of any instrument-assisted delivery and do not indicate severe birthing trauma sufficient to cause corneal perforation [2].

Other aspects of this case which also make birth trauma a less likely explanation for this neonate's corneal perforation was the presence of unilateral bupthalmos, persisting corneal edema and an elevated intraocular pressure. These findings are more in keeping with a diagnosis of primary or secondary congenital glaucoma [3].

Primary congenital glaucoma is due to abnormal development of the ocular outflow drainage system - the trabecular meshwork. It is most commonly bilateral in $75 \%$ of cases and occurs in approximately $0.05 \%$ of children often diagnosed during their first year of life. Secondary glaucomas can occur due a variety of other ocular and systemic causes (Table 2).

With the benefit of hindsight, early referral of this neonate's corneal haze and an exploration of the right eye under anesthesia soon after delivery may have assisted with this diagnosis. Typical findings in congenital glaucoma include: a corneal diameter in a neonate greater than $9.5 \mathrm{~mm}$, breaks in Decemet's membrane-Haab's straie, intraocular pressure above $21 \mathrm{mmgh}$ and visible cupping of optic nerve. In addition, findings in the anterior segment such as total or partial loss of the iris (aniridia) or abnormal adhesion of the iris or lens in part to the cornea (Peter's anomaly) can also co-exist leading to secondary congenital glaucoma. Some of these findings were present in the histopathological specimen of this enucleated eye, suggesting that this eye was likely to have had a degree of anterior segment malformation in-utero [4] [5].

This case was further complicated by a suspect retinal detachment. Whether this was infact a true detachment or remnants of peristant hyaloid vasculature in a premature neonate is difficult to conclude on ultrasound alone. In general posterior segment persistent hyaloid vasculature arises from the disc and extends to the posterior lens capsule and may be associated with retinal detachment [6]. Surgical intervention to excise membranes has been attempted in some cases although runs the risk of multiple re-operations. In general these cases are conservatively managed [7].

The histopathology from the enucleated eye in this case has proven to be the key to the likely etiology of this unusual presentation. It has been postulated that structurally malformed eyes are more prone to trauma at birth. [8].

A proposed sequence of events in this case may have been that a rise in intraocular pressure due to a congenitally malformed anterior eye segment, gradually progressed in utero, resulting in diffuse corneal decompensation 
Table 1. Causes of corneal oedema in a neonate.

\begin{tabular}{|c|c|}
\hline $\begin{array}{l}\text { Trauma } \\
\text { Infection }\end{array}$ & Instrument assisted delivery \\
\hline Congenital & $\begin{array}{l}\text { Glaucoma } \\
\text { corneal dystrophies } \\
\text { Mucopolysaccharidoses } \\
\text { Anterior segment malformation: } \\
\text { Peter's anomaly, Axenfeld-Reiger's }\end{array}$ \\
\hline Other & Dermoid \\
\hline
\end{tabular}

Table 2. Classification of congenital glaucoma.

Primary infantile Glaucoma
Secondary Glaucoma
$\begin{aligned} & \text { 1) Angle anomalies } \\ & \text {-Sturge-Weber syndrome } \\ & \text {-Anirida } \\ & \text {-Anterior segment dysgenesis } \\ & \text { 2) Posterior segment anomalies } \\ & \text { 3) Aphakia } \\ & \text { 4) Intraocular inflammation } \\ & \text { 5) Tumors } \\ & \text { 6) Trauma }\end{aligned}$

and subsequent corneal perforation. Birth trauma though initially suspected as the primary cause for the perforation may in reality have had a minor, if any effect in the overall sequence of events.

\section{Conclusion}

Instrument-assisted delivery causing corneal perforation is a rare occurrence. Any presentation of suspected corneal injury following instrument-assisted delivery should improve shortly after birth and persisting signs should raise the suspicion of another cause. Worsening corneal edema and bupthalmos is suspicious for congenital glaucoma and requires prompt exploration under anesthesia. Early surgical intervention for control of elevated intraocular pressure may improve the likelihood for visual recovery and preservation of the eye.

\section{References}

[1] Lam, F. and Batt, P. (1997) Spontaneous Corneal Perforation in Premature Infants. Singapore Medical Journal, 38, 469470.

[2] Johanson, R. and Menon, V. (2005) Vacuum Extraction versus Forceps for Assisted Vaginal Delivery. Cochrane Database of Systematic Reviews, 2, Article ID: CD00224.

[3] Honig, M. and Barraquer, J. (1996) Forceps and Vacuum Injuries to the Cornea (Histopathologic Features of Twelve Cases and Review of the Literature). Cornea, 15, 463-472. http://dx.doi.org/10.1097/00003226-199609000-00005

[4] Tawara, A and Inomata, H. (1984) Developmental Immaturity of the Trabecular Meshwork in Juvenile Glaucoma. American Journal of Ophthalmology, 98, 82-83. http://dx.doi.org/10.1016/0002-9394(84)90193-4

[5] Beck, A. (2001) Diagnosis and Management of Pediatric Glaucoma. Ophthalmology Clinics of North America, $14,1501$.

[6] Dass, A. and Trese, M. (1999) Surgical Results of Persistent Hyperplastic Primary Vitreous. Ophthalmology, 106, 280284. http://dx.doi.org/10.1016/S0161-6420(99)90066-0

[7] Alexandrakis, G. and Scott, I. (2000) Visual Acuity Outcomes with and without Surgery in Patients with Persistent Fetal Vasculature. Ophthalmology, 102, 106-108.

[8] William, W. and Hay, M. (2004) Current Pediatric Diagnosis and Treatment. 15th Edition, McGraw-Hill Prof Med/Tech. 
Scientific Research Publishing (SCIRP) is one of the largest Open Access journal publishers. It is currently publishing more than 200 open access, online, peer-reviewed journals covering a wide range of academic disciplines. SCIRP serves the worldwide academic communities and contributes to the progress and application of science with its publication.

Other selected journals from SCIRP are listed as below. Submit your manuscript to us via either submit@scirp.org or Online Submission Portal.
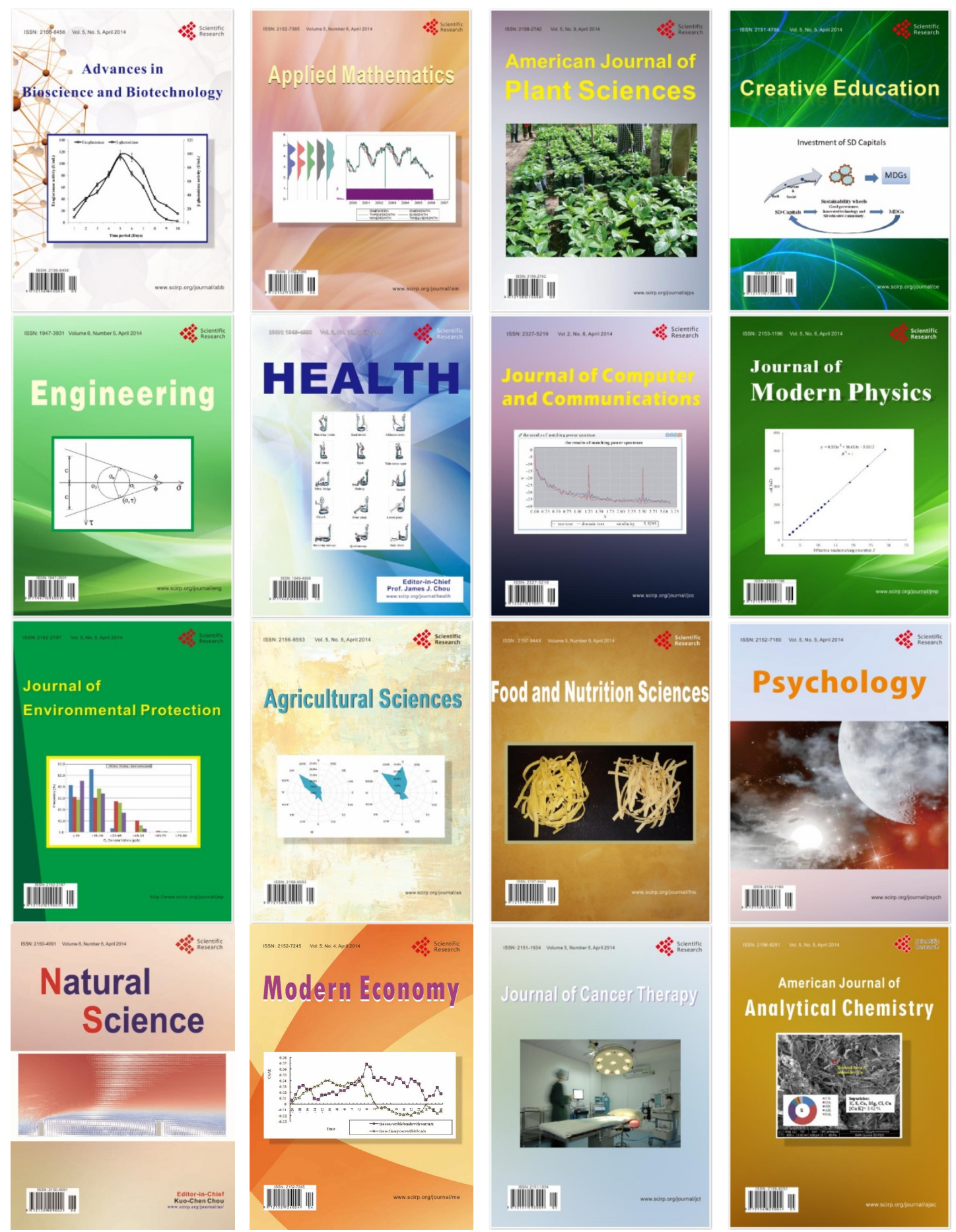